\title{
What Should be Prescribed for Patients with Suspected Multidrug- Resistant Tuberculosis in the Absence of Drug Sensivity Results
}

\author{
Batool Sharifi-Mood ${ }^{1, *}$ \\ ${ }^{1}$ Infectious Diseases and Tropical Medicine Research Center, Zahedan University of Medical Sciences, Zahedan, Iran \\ "Corresponding author: Batool Sharifi-Mood. MD, MPH, Zahedan University of Medical Sciences, Zahedan, Iran. Tel: +98-5433228101-2, Fax: +98-543 3236722, E-mail: \\ batoolsharifimood@yahoo.com
}

Received 2016 December 02; Accepted 2016 December 14.

Tuberculosis (TB) is one of the most important preventable diseases worldwide. Every year, 8 million new cases of TB are reported by WHO (world health organization). Case finding, treatment, and monitoring are the 3 major factors that prevent morbidity, mortality, and occurrence of drug resistance (1). Multidrug-resistant tuberculosis or MDR -TB is a form of tuberculosis, caused by mycobacterium tuberculosis organism, that is resistant to isoniazid (INH) and rifampin (RIF) $(1,2)$. It is reported that $5 \%$ of patients with tuberculosis have MDRTB. There are various reasons for this phenomenon including adverse drug reaction, failure to complete treatment, cost of treatment, stigma about TB, and disease mismanagement (1-3). Nonetheless, other important factors are delay in treatment and genetic factors (4). Treatment of MDR-TB is very difficult, especially in patients with HIV, pregnant women, and children. Inappropriate treatment and mismanagement can lead to life-threatening disease and death $(3,5)$. MDR- TB should be treated and managed by an experienced physician. Drug susceptibility of mycobacterium tuberculosis can be defined by DNA-based method or culture, however, sometimes, conducting tests to prove drug- resistant TB can take weeks $(1,3,6)$. Therefore, physicians should start treatment with an empirical regimen as soon as possible when they are faced with a suspected case of multidrug-resistant TB. Then, when the test results are out, the treatment regimen should be adjusted according to the results (3, 5-7). Selecting drugs to treat MDR-TB should be done based on drug susceptibility results and TB drug resistance patterns in each region. This regimen should be a combination of secondline anti-TB drugs that are more toxic than first line anti -TB drugs and should be used for longer durations (at least 20 months). WHO guideline recommends using at least 4 second-line drugs including (an injectable drug) kanamycin, a new fluoroquinolone (levofloxacin, moxifloxacin), prothionamide, and para-aminosalicylic acid or cycloserine when the physician starts the empirical therapy and does not know the results yet $(3,5,7)$. In addition to pyrazinamide, fluoroquinolones (such as moxifloxacin and levofloxacin) are the most effective second-line drugs for MDR-TB. Despite the few reports of MDR-TB, which are also resistant to these agents, linozolid is recommended in cases of fluoroquinolone-resistant MDR-TB (5-8). Thus, it is of importance that these patients be monitored closely throughout treatment. Directly observed therapy (DOT) should be used in the treatment of drug-resistant TB to ensure adherence and prevention of changing MDR -TB to XDR-TB. In conclusion, it is recommended that patients with MDR-TB refer to physicians experienced at treating this disease.

\section{Acknowledgments}

There is no acknowledgment.

\section{Footnotes}

Authors' Contribution: Batool Sharifi-Mood wrote the paper.

Financial Disclosure: There was no conflict of interest. Funding/Support: There was no financial support.

\section{References}

1. Metanat M, Sharifi-Mood B, Shahreki S, Dawoudi SH. Prevalence of multidrug-resistant and extensively drug-resistant tuberculosis in patients with pulmonary tuberculosis in zahedan, southeastern iran. Iran Red Crescent Med J. 2012;14(1):53-5. [PubMed: 22737557].

2. Yew WW. Management of multidrug-resistant tuberculosis and extensively drug-resistant tuberculosis: current status and future prospects. Kekkaku. 2011;86(1):9-16. [PubMed: 21401001].

3. World Health Organization . Guidelines for the programmatic management of drug-resistant tuberculosis. Geneva: Switzerland; 2008.

4. Hashemi M, Eskandari-Nasab E, Moazeni-Roodi A, Naderi M, SharifiMood B, Taheri M. Association of $<\mathrm{I}>\mathrm{CTSZ}<\mid \mathrm{I}>$ rs34069356 and $<$ I $>$ MC3R $<$ I $>$ rs6127698 gene polymorphisms with pulmonary tuberculosis. Int J Tuberc Lung Dis. 2013;17(9):1224-8. doi: 10.5588/ijtld.12.0762.

5. Caminero JA, Sotgiu G, Zumla A, Migliori GB. Best drug treatment for multidrug-resistant and extensively drug-resistant tuberculosis. Lancet Infect Dis. 2010;10(9):621-9. doi: 10.1016/S1473-3099(10)70139-0. [PubMed: 20797644].

6. World Health Organization. The use of molecular line probe assay for the detection of resistnace to second-line anti-tuberculosis drugs.; 2013. 
7. Yew WW, Chan CK, Leung CC, Chau CH, Tam CM, Wong PC, et al. Comparative roles of levofloxacin and ofloxacin in the treatment of multidrugresistant tuberculosis: preliminary results of a retrospective study from Hong Kong. Chest. 2003;124(4):1476-81. [PubMed:14555582].
8. Jo KW, Lee SD, Kim WS, Kim DS, Shim TS. Treatment outcomes and moxifloxacin susceptibility in ofloxacin-resistant multidrug-resistant tuberculosis. Int J Tuberc Lung Dis. 2014;18(1):39-43. 